\title{
WLGauge - A Web Link Gauge to Measure the Quality of External WWW Links
}

\author{
Omar Garcia, Srivalli Vilapakkam Nagarajan, and Peter Croll \\ Centre for Dependable Software Engineering and Training (CDSET) \\ School of IT and Computer Science (SITACS) \\ University of Wollongong Australia \\ \{omar, svn95, croll\} @uow.edu.au
}

\begin{abstract}
External Broken links have a negative impact on any web page user. However, little or no information is provided in advance to advise the user of the quality of the available links before they hit them. Our contributions in this paper are: (1) To present our arguments to suggest that the external link quality is not binary (broken or available) but instead it is a continuous value from low reliability to high reliability (2) To emphasise the need for the web designers to provide some information in advance to the user suggesting the quality of the link. This approach is very similar to having the image size next to a potential loading image giving the user a broad idea about the resources needed to download that image. (3) To present a new tool called WLGauge that can measure some quality attributes of the external links in a web page. WLGauge is an open framelet that can be easily plugged on to any web site development toolbox. WLGauge has been designed with an intention to help the web designer and web maintenance teams to understand as well as improve the quality of their web pages.
\end{abstract}

Keywords: Website, open framelets, external link quality, website quality and automatic testing.

\section{Introduction}

Websites attracts audience from all corners of the world. Web users are increasingly annoyed by the poor quality and performance of some web sites. Poor web site quality, performance or accessibility is potentially dangerous to business operations in today's competitive market. Because of this reason, Web Engineering has attracted more attention in recent years than ever before. Nowadays, much of the effort is put into producing websites that are not only good in quality but also are reliable at all times. Therefore, reliability (or accessibility) and quality have been the two main factors in deciding the web user's satisfaction. Link rot is one of the many challenges facing the web community. Link rot is a situation in which the decay of the www links occurs as the sites they connect to changes or disappears [1]. Link rot has a direct impact on the website usability [2]. The objective of this paper is two fold. Firstly, we emphasise that the link rot problem is not binary but instead falls within a range of 
quality levels from low to high reliability. Secondly, we suggest that the open framelets is a viable technique to build and test the web software for external broken links.

In section 2 we provide a background to the current situation and identify the problems surrounding the web community. The external broken links problem has been analysed and a solution to handle them has been proposed. Section 3 discusses the related work done by other software companies/developers to handle the broken external links and related problems. Section 4 highlights our contribution on the development of a Web Link Meter called "WLGauge" which is an open framelet capable of measuring the quality of the external links. In section 5 the WLGauge design is described in detail. Section 6 will discuss the work done in the evaluation of the WLGauge and its performance. In section 7 potential areas for future work are identified and our contributions and conclusions are highlighted.

\section{Background to the Link Rot Problem}

According to GVU [2] slow speed of Internet access $(66.1 \%)$ and the proliferation of the broken links $(49.90 \%)$ are the main problems facing today's Web users. In order to improve the speed of the service, faster links and data compression algorithms are widely used. The broken link problem is seen as a manifestation of a more general problem known as link rot. As mentioned earlier, link rot can be defined as the decay of World Wide Web links since the sites that they connect to change or disappear. This is a huge problem, as it cannot be easily brought under control in any way. The web designer does not have control over these links. On top of all this, link rot now seems to be a fairly common problem. It has been recently reported in [3] that at least about $30 \%$ of all the web pages experience link rot.

Professors Brooks and Markwell, from the University of Nebraska-Lincoln, compared the rate of link rot to the type of "extinction equation" commonly used to describe natural processes such as radioactive decay [4]. According to them, the hyperlinks in their study had an expected "half-life" of 55 months. Also, the extinction rate was dependant on the domain. For example, '.edu' has the biggest loss of all the domains.

The World Wide Web is constantly changing. While dynamicity is a certainty in web the lesson to learn from link rot is to regularly check the status of all the links used in your websites. It is even more important with online education (e-learning) to make sure that all the links work properly. This should be the norm in preparing any course that relies on Internet resources beyond our control [5]. The advice is to detect and correct the rotten links before they destroy you. 


\subsection{A Closer Look at the Problem and a Proposed Solution}

The problem of link rot is not binary (broken or not broken). There may be many reasons why some of the links are not available at a particular time. Some of the common reasons are - server down, links down and faulty router. It is worth noting that not all websites work 24 hours a day. However, in order to give a sensible measure of the link quality, frequent testing and recording of the response time(s) as well as the behaviour of the external links in a web site must be stored in a test profile. This profile will provide information to generate a quality measure of the link. Sampling can be done on a timely basis. The user can make a decision on when and how often sampling should be done.

External links are those WWW links over which we have no control. Some external links are not available all the time. Few others are always available. Therefore there is a range here with always available on one end and not available at all at the other end. It will be extremely helpful if the quality of the link can be measured and then used to determine where the link falls in the range. The clients can then be informed about the information on the quality of the link before they hit the link. The following measures will provide web users with valuable information on the quality of the link:

- Percentage of success versus failure: It is a measure to find out the percentage of time when the link's accessibility works (or is successful) or not. This measure will provide an indication of the reliability and accessibility of the websites.

- Average time to respond: This is the average time required to retrieve the information from an external link. This measure will give an indication of the speed and performance of the website after hitting the link. Response time and latency parameters will help to understand end-to-end response time as well as to check if there are parts of a site that are very slow in comparison to the other parts.

- Date of last successful hit: It becomes easy to look at the history and tell if the link has been accessed in the recent past. This information will help the user know about the status of the link.

In the current world, developing tools to automatically measure and to collect the above information for the web users has become a crucial matter in meeting the user's needs. Therefore, an open framelet called "WLGauge" was developed to help the webmasters, web application developers and the web site quality assurance managers to collect the above useful data so that quality related information can be provided to their clients. WLGauge is capable of measuring the quality of the external links and generating a profile of it, which in turn can be used to either provide a report that can be forwarded automatically to the clients in a simple manner or be able to dynamically update the web page to show a visual clue of the link quality. 


\section{Related Work}

A variety of products are available to alleviate the link rot problem and they can be classified in to two groups:

- Tools that are able to test a link or group of links and generate a report with the status of the link among other things. Example: Tetranet LinkBot [6] and NetMechanics toolbox [7]; also freeware or shareware like CheckBot [8] and Xenu's Link Sleuth [9]

- A service provider (a company) that on a timely basis checks the client's website and informs them if the website has problem like broken links and others. Example: Link Alarm [10] and LinkWalker [11] belong to this group.

Table 1 lists a few of the products available and their characteristics.

Table 1. Software to check broken links

\begin{tabular}{|c|c|}
\hline Product Name & Features \\
\hline Link Walker & $\begin{array}{l}\text { - } \quad \text { Verifies the external links for access } \\
\text { - } \quad \text { Scans sites daily to check for broken links } \\
\text { - } \quad \text { Lets the client know when an inbound link is broken }\end{array}$ \\
\hline $\begin{array}{l}\text { Xenu's Link } \\
\text { Sleuth }\end{array}$ & $\begin{array}{l}\text { - Link verification covers all the links, images, frames, } \\
\text { plug-ins, backgrounds; local image maps scripts and java } \\
\text { applets. } \\
\text { - } \quad \text { Displays a updated list of URLs } \\
\text { - } \quad \text { Free and has a simple user interface }\end{array}$ \\
\hline Checkbot & $\begin{array}{l}\text { - Link verification on a set of HTML pages } \\
\text { - Can check for single or more documents on one or more } \\
\text { server } \\
\text { - Creates a report which summarises all the links with } \\
\text { errors }\end{array}$ \\
\hline Link Alarm & $\begin{array}{l}\text { - Online web service that checks every link on every page } \\
\text { of the client's site } \\
\text { - } \quad \text { Confidential reports are sent about any problems } \\
\text { - } \quad \text { Link failure is classified according to the type of defect } \\
\text { - } \quad \text { LinkAlarm rating for each check is generated }\end{array}$ \\
\hline
\end{tabular}

Difference between the other products and WLGauge: First, as stated before, all these product/services consider the link rot problem as binary and therefore do not 
provide a history of tests whereas WLGauge is able to provide a history of the test results. Secondly, the current tools and services do not provide any automatic ways to fix the problem or to provide a graph or textual clue of the quality of the link to the web user. It is up to the web manager to feed this information on his web site.

\section{WLGauge - An Open Framelet}

\subsection{Framelets}

Pree and Koskimies first introduced the word framelet in [12] and later reinforced in [13], where they defined framelets as mini frameworks) that have the following characteristics:

- $\quad$ They are small in size (less than 10 classes);

- They do not assume main control of an application;

- They have a clearly defined interface

A framework is a design of a reusable application or a part of an application represented by a group of classes in such a way that they collaborate with each other.

\subsection{Open Source}

One of the ongoing impediments to increase software reuse has been the concern experienced by the developers when the source code is not available to them. Even when the developers do not need to modify the source code, there appears to be a psychological problem whenever the source code is not available. The lack of source code is perceived as a potential threat that hinders the reuse of somebody else's code. We are aware that software developers may face potential economic threats by delivering open source products, but we still embrace the open source movement because the benefits to the development community must prevail over the benefits to individual component developers. For a more detailed discussion on open community see $[14,15]$. We consider WLGauge as an open framelet because it is both a mini framework (satisfying the definition of framelets) and is free software for anyone to use.

\section{WLGauge Design}

One of the goals of the WLGauge design is to make the system as automated as possible. This means reduced human interaction and reduced amount of work to be done due to the use of automatic testing tools [16].

Figure 1 shows the operations involved in the usage of WLGauge. The main processes involved in the design of WLGauge are: 
1. URLCollector: Collecting the external url from the web page and storing them in a file. This process can be done manually or automatically. A simple program to achieve this comes with the framelet.

2. URLChecker: This process will run basically an http request to the external link and collect the data in a file or database. It basically checks all links in the file generated in the previous step. It does only one test on the link and appends the information to the test profile records or create a new test profile if it does not exist.

3. WebPageMechanic: This process will transform the http pages in a java servlet page by adding graph or textual information next to the external links. The following is a java scriptlet that will be inserted in a web page and will show the success ratio of an external link.

$<\%=$ new WLGauge ("www.uow.edu.au").getSuccessRatio(). \%>

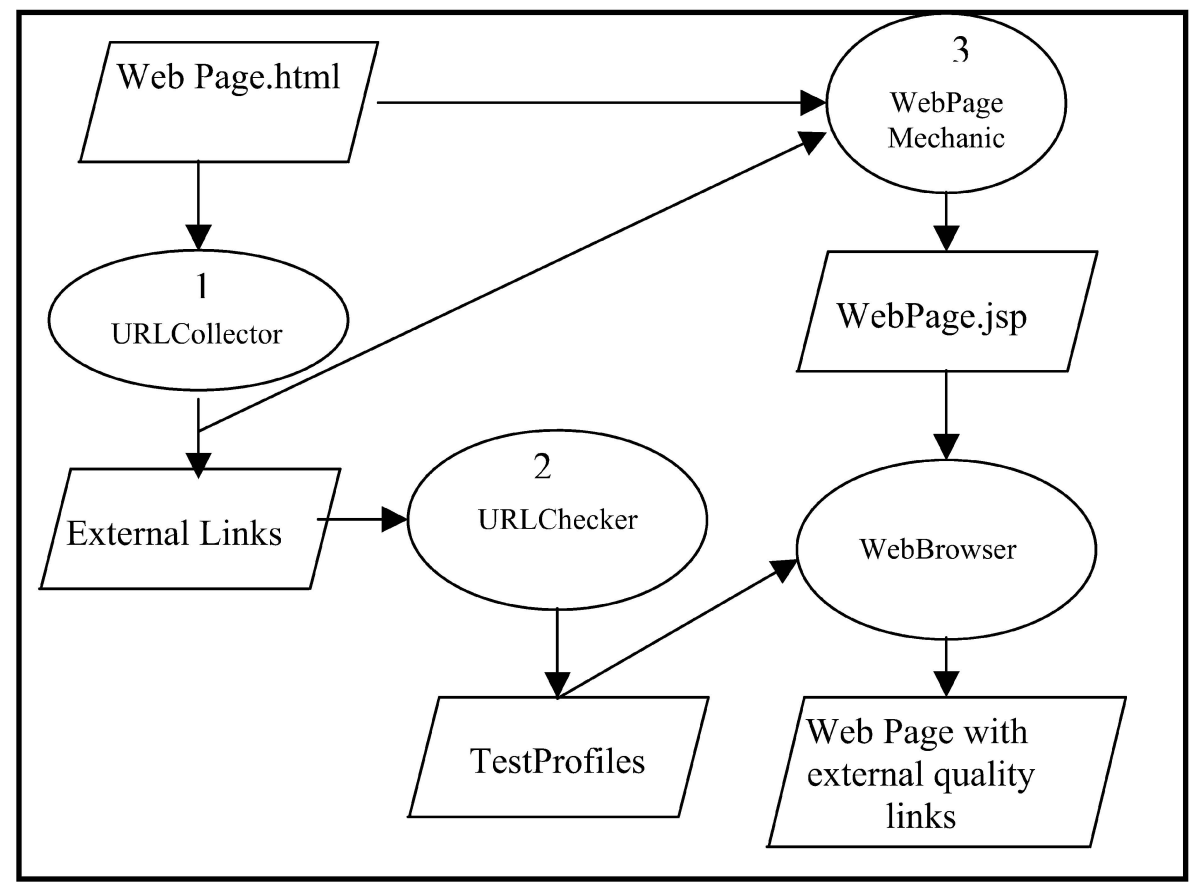

Fig. 1. WLGauge Operation

The class diagram for WLGauge framelet is shown in figure 2. 


\begin{tabular}{|l|l|l|}
\hline WLGauge & & TestSample \\
$\begin{array}{l}\text { ID } \\
\text { URL } \\
\text { TestSamples }\end{array}$ & $\begin{array}{l}\text { StartDate } \\
\text { stopDate } \\
\text { isOK } \\
\text { returnCode }\end{array}$ \\
\cline { 2 - 3 } $\begin{array}{l}\text { getAverageResponseTime() } \\
\text { getSuccessRatio() } \\
\text { getDateLastTest() } \\
\text { getDateLastTestOK() } \\
\text { getNumberOfTests() }\end{array}$ & $\begin{array}{l}\text { getStartDate() } \\
\text { getStopTime() } \\
\text { getElapseTime() }\end{array}$ \\
\hline
\end{tabular}

Fig. 2. WLGauge Class Diagram

Basically, the instance variables for the class WLGauge are the URL for the external link, an internal ID for control purpose and the set of test samples. The methods give us the average response time in milliseconds, the success ratio (a value between 1 and 0 ), the date of the last test and the number of tests performed on that link. One instance of the class TestSample is created every time a test is run. The instance variables and methods are pretty straightforward things that can be understood easily. An example explaining the use of WLGauge tool is demonstrated as follows. Figure 3 shows a web page for a software engineering page. This is the original html page. Figure 4 shows the web page generated by the system after the WLGauge has been used. It can be noted that the difference between the content in webpage in figure 3 and 4 is the addition of the average response time information next to the links in the figure 4. A color representation can be used but it is not used in this example. Also, in figure 4 a note is placed at the bottom of the page indicating what the value next to the link means to the user (in this case it is the average response time to the link).

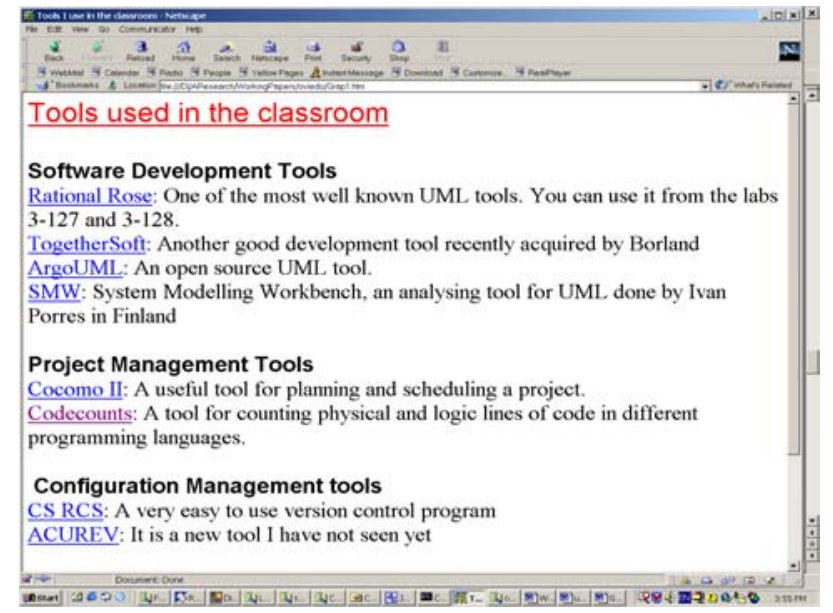

Fig. 3. An original html page 


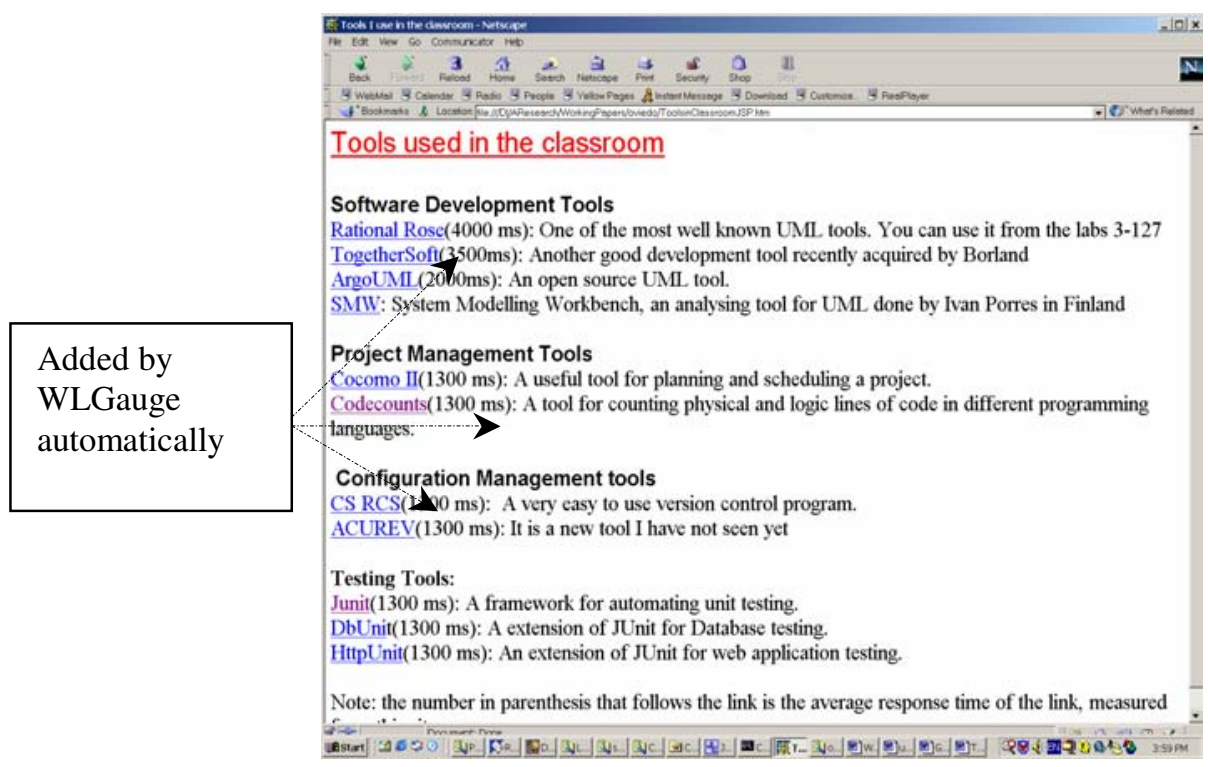

Fig. 4. A modified web page generated with the use of WLGauge

The WLGauge software is available at www.uow.edu.au/ omar/WLGauge.

\section{Evaluation Work Done on WLGauge}

In this section an evaluation of the tool developed is provided. WLGauge has a very limited functionality, for example it has only the skeletal framework and the tester/user has to provide the code for testing for the sampling scheme (information like when the test has to be carried out, test dates, timings etc have to be supplied to the existing framework).

The main question is how to report the quality of the link measured?

- A graphical representation based on different colours like green, yellow or red.

- A textual value, for example: the average time to access the link, the ratio of success.

- A hover over the link that will display some information about the quality of the link.

- A sound, which conveys some sort of guiding information about the quality of the link.

To provide the above functionalities one must devote thousands of programming hours. This means increasing costs for the product and fattening the software [17]. However the reality is that a typical user will not choose all these features. Therefore, framelets provide a blueprint where additional features are easily plugged when they are really needed. 
The quality metrics measured are very much dependent on the performance characteristics of the web server client from where it is measured [18]. When the WLGauge program runs its tests on the links, the links are solved from the web client, so there is margin of error on this. To be more realistic the quality of the link should be measured on the client. Technically it is possible, TestRunner and PageUpdate has to be moved to the client. This depends on the answers to the questions like - does the user want to do this? And does the user know in advance the links they will be going through? We experimented with the tool to collect the response times for three cases: EAFIT university link, Microsoft link and University of Wollongong link. As expected the response times for our own University of Wollongong link was quicker than the response times for the other two because of the proximity of the server that was accessed. Microsoft was a large commercial company and they had better response time compared to the response time of the EAFIT university link.

In table 2, below we highlight the main advantages and limitations of WLGauge.

Table 2. WLGauge advantages and limitations

\begin{tabular}{|c|c|}
\hline Advantages of WLGauge & Disadvantages of WLGauge \\
\hline Small and Reduced complexity & Too simple \\
\hline $\begin{array}{c}\text { Easy to learn and use } \\
\text { Easy to plug onto the wed designer/manager's } \\
\text { tools }\end{array}$ & $\begin{array}{l}\text { It does not support some additional features } \\
\text { available in other products }\end{array}$ \\
\hline Open framelet & Not much support offered \\
\hline $\begin{array}{c}\text { It uses Java as the language for implementation } \\
\text { (Java offers platform-independence, designed for } \\
\text { internetworking, portability and security) }\end{array}$ & $\begin{array}{c}\text { It uses Java as the language for implementation } \\
\text { (interpreted Java programs runs slower than the } \\
\text { compiled programs) }\end{array}$ \\
\hline
\end{tabular}

\section{Future Work and Conclusions}

\subsection{Future Work to Be Done}

WLGauge can be made slimmer by not using the HttpUnit framework [20] and by directly using java.net, we have not done this yet mainly because it will increase the development time. HttpUnit is another popular framework for testing based on Junit [21] with the purpose of testing web applications, specifically, to simulate certain functionalities of the browser with the purpose of examining the result of a web page. It is important not to add complexity to the existing framework by adding unwanted features and clearly draw the difference between indispensable and luxurious features.

WLGauge has to be used extensively before its usefulness can be completely understood. Therefore it will have to be tested in the field for a long term before determining how easy it is to work with.

In the future, to move the URLChecker to the client (instead of the server) will be explored. A similar idea will be to rewrite the WLGauge system as a browser plug in. 
More effort must be put into finding answers for some of the following questions: Is it worth the cost of tracing the links? And what are the challenges in doing so ? (privacy, ethical, etc).

Based on the results of our experimentation and the feedback collected from the user experiences, the WLGauge framelet will be continuously improved. A multithreaded version of WLGauge will also be explored. A multi-threaded option can be useful where more than one link is checked at the same time. This idea was not incorporated in the first version because of one important user behaviour - very often, users will go one link at a time and the test will reflect the way users operate.

\subsection{Conclusions}

Our contribution to this paper includes identification of the link rot problem as non binary problem and that the quality of the external links should be measured so that the quality of the web sites can be understood. WLGauge was specifically developed to let users know the crucial information on the quality of the external links on their websites. According to [22] automated website quality testing is both an opportunity and a challenge.

Watchfire [23] tell us that $58 \%$ of the consumers said that if the site failed on their first visit, they would never return to it. Jakob Nielsen [24] says that broken links annoy the users and deprive them of the value they would have gotten from the site. In our opinion simple framelets like WLGauge have a huge potential to perform very well for small-scale web applications and we certainly believe that in the years to come everyone will realise how crucial such simple tools are in meeting the web user's expectations.

Acknowledgment. We appreciate the comments and value suggestions of my friends and colleagues Lourdes de Miguel Garcia and Khin Than Win.

\section{References}

[1] The Jargon Dictionary at http://info.astrian.net/jargon/ dated 19/01/2003

[2] Graphic, Visualization, \& Usability Center's (GVU) 7th WWW User Survey at http://www.cc.gatech.edu/gvu/user_surveys/survey-1997-04/ dated 19/01/2003

[3] Evans, M P. Furnell, S M., "The Resource Locator service: Fixing a law in the web", The International Journal of Distributed Informatique, v 37 n3-4 Nov 5 2001, Computer NetWorks.

[4] Markwell, J. Brooks, D.W. "Broken links: The ephemeral nature of educational WWW hyperlinks", Journal of Science Education and Technology, June 2002

[5] Rumsey, M. "Runaway Train: Problems of Permanence, Accessibility, and Stability in the Use of Web Sources in law Review Citations", Law Library Journal vol 94:1

[6] Link Bot at http://www.watchfire.com/ dated 20/01/2003

[7] Toolbox at http://www.netmechanic.com/ dated 19/01/2003

[8] Checkbot at http://degraaff.org/checkbot/ dated 19/01/2003 
[9] Xenu at http://home.snafu.de/tilman/xenulink.html dated 20/01/2003

[10] Link Alarm at http://www.linkalarm.com/ dated 20/01/2003

[11] Link Walker at http://www.seventwentyfour.com/ dated 19/01/2003

[12] Pree, W. Koskimies, K. "Rearchitecturing Legacy Systems-Concepts \& Case Study", First Working IFIP Conference on Software Architecture (WICSA), San Antonio, Texas, February 1999

[13] Pree, W. Koskimies, K. "Framelets small is beautiful", in Building Application Frameworks: Object-Oriented Foundations of Framework Design, Wiley \& Sons, New York City, 2000

[14] Newman, N. "The Origins and Future of Open Source Software", at http://www.netaction.org/opensrc/future/oss-whole.html

[15] Gabriel, R. "The Feyerabend Project", at http://www.dreamsongs.com/Projects.html, dated 20/01/2003

[16] Garcia, O. "Automatic Software Testing in O.O. Languages", Proceedings of SigSoft 2001, Encuentro Iberoamericano, Universidad Distrital, August 29-31, 2001 Bogota, Colombia.

[17] Shin, D. Koh, K. Won, Y. "Measurement and Analysis of Web Server Response Time", Proc. of APCC 2000(Asia Pacific Conference on Communications), Seoul, Korea, Oct. 2000.

[18] Wirth, N. “A plea for lean software”, IEEE Computer, Volume: 28 Issue: 2, Feb 1995 pp: 64-68

[19] http://www.wired.com/news/business/0,1367,10251,00.html dated 18/01/2003

[20] HttpUnit at http://sourceforge/ dated 19/01/2003

[21] JUnit at http://junit.org/ dated 19/01/2003

[22] The WebSite Quality Challenge, at http://www.soft.com/eValid/Technology/White.Papers/ dated 19/01/2003

[23] WebSite Quality Matters, at http://www.watchfire.com/solutions/quality.asp dated 18/01/ 2003

[24] Jakob Nielsen, "Fighting linkrot “, at http://www.useit.com/alertbox/, dated 18/01/ 